\title{
9 Innovation and Diversification Via Plasticity-Led Evolution
}

\author{
Nicholas A. Levis \\ Indiana University \\ David W. Pfennig \\ University of North Carolina
}

\section{CONTENTS}

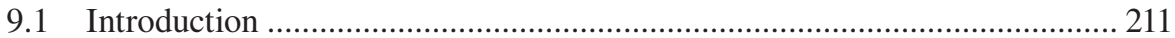

9.2 Plasticity-Led Evolution: Historical and Contemporary Perspectives ......... 212

9.3 How Plasticity-Led Evolution Promotes Innovation and Diversification ..... 215

9.3.1 Plasticity-Led Evolution and Innovation ………............................ 215

9.3.2 Plasticity-Led Evolution and Diversification .................................... 218

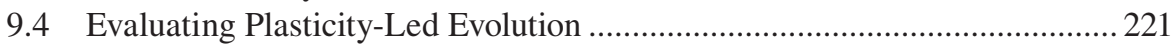

9.5 Plasticity-Led Evolution: Consensus, Controversy, and Challenges .............222

9.5.1 Plasticity-Led Evolution: Where Is the Consensus? ...........................223

9.5.2 Plasticity-Led Evolution: Where Is the Controversy?........................228

9.5.3 Plasticity-Led Evolution: Where Are the Challenges? ........................229

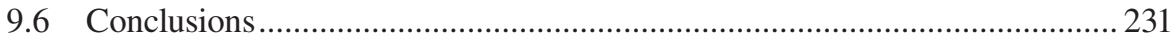

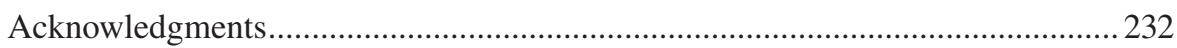

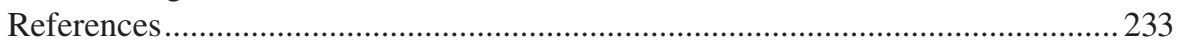

\subsection{INTRODUCTION}

Biodiversity requires explanation. Why are there so many different kinds of living things, and where do their unique features come from? Historically, biologists have attempted to address these questions by focusing on the ecological and genetic factors that foster evolutionary diversification and innovation. By contrast, less attention has been paid to development. Yet, a key feature of development-its tendency to be adaptively responsive to changes in an organism's environment—might play a critical role in the origins of new traits and new species.

In this chapter, we explore these issues by introducing the concept of plasticityled evolution-adaptive evolution that is set in motion by phenotypic plasticity. As we describe, there are numerous ways that plasticity can contribute to the origins 
of new traits and new species, suggesting that plasticity-led evolution may play a largely underappreciated role in driving evolutionary innovation and diversification. We then briefly discuss how to test for plasticity-led evolution in natural populations and summarize its empirical support. Finally, we consider where the study of plasticity-led evolution currently stands. In doing so, we highlight both areas in which a consensus has been reached and areas in which controversy remains. We close our chapter by providing answers to some commonly asked questions about plasticityled evolution and offering suggestions for future research.

We begin, however, by placing the study of plasticity-led evolution in a historical context before providing a modern description of the concept.

\subsection{PLASTICITY-LED EVOLUTION: HISTORICAL AND CONTEMPORARY PERSPECTIVES}

Even before the term 'phenotypic plasticity' was coined in 1914 by the Swedish biologist Herman Nilsson-Ehle, biologists recognized that a change in the environment could trigger pronounced variation (that is, diversity) within species. For example, 'polyphenism'- the occurrence of alternative environmentally induced phenotypes within the same population (Mayr 1963; Nijhout 2003; West-Eberhard 2003) fascinated many of the early evolutionists, such as August Weismann, Conrad Waddington, and Richard Goldschmidt. As they and others since came to appreciate, these environmentally induced morphs can be as different in behavior, morphology, physiology, and/or life history as different species (see Figure 9.1). Thus, an important question arose: were the forces that generate such diversity within species the same as those that generate diversity between species? Weismann (1882), for example, was convinced that the study of polyphenism was key to understanding species differences (see the Preface of this volume).

At the same time, biologists began to ask whether environmentally induced changes to an organism's behavior preceded and facilitated evolutionary change in morphology (e.g., Lamarck 1809; Baldwin 1896; Morgan 1896; Osborn 1896). Such a change in morphology could arise, the argument went, if a new behavior altered the selective pressures an organism experienced (e.g., by subjecting it to a new food source or competitive environment), and/or if the new behavior itself brought about morphological changes that were somehow inherited (for details on how this process might occur, see Bonduriansky and Day 2018). In either case, the change in behavior could cause the organism's genes to be 'pulled' into the next generation, thereby creating evolutionary change (see also Chenard and Duckworth 2021 in this volume).

Behaviors are not the only phenotypes that are environmentally responsive. Indeed, during the mid-twentieth century, researchers proposed that environmentally initiated change in diverse morphological and physiological traits could precede and facilitate genetic change (e.g., Schmalhausen 1949; Waddington 1953; Bateman 1959; see Box 3.2 in Pfennig 2021 in this volume). However, much of this work was ignored or dismissed by most evolutionary biologists (Simpson 1953a; Williams 1966; Orr 1999; see also Futuyma 2021; Pfennig 2021). 
(a)

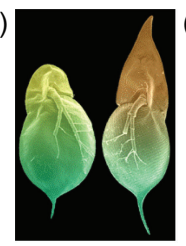

(d)

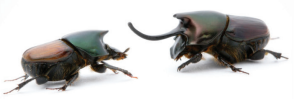

(b)

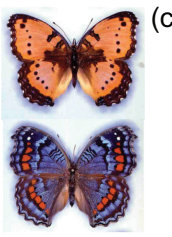

(e)

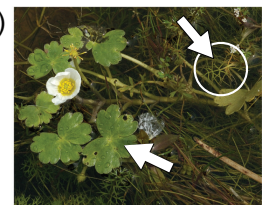

FIGURE 9.1 Phenotypic plasticity can generate diversity within species that is sometimes as great (or greater) than that normally seen between different species. To illustrate this point, we show examples of environmentally induced alternative phenotypes ('polyphenism'). (a) Normal (left) and predator-induced (right) morphs of water fleas, Daphnia cucullata; (b) wet-season (top) and dry-season (bottom) gaudy commodore butterflies, Precis octavia; (c) omnivore (top) and carnivore-morph (bottom) spadefoot toad tadpoles, Spea multiplicata; (d) small-horned (left) and large-horned (right) dung beetles, Onthophagus nigriventris; (e) broad, aerial leaves and narrow, submerged leaves (circled) on the same water crowfoot plant, Ranunculus aquatilis. (Reproduced from Pfennig et al. [2010], with the kind permission of the publisher.)

A key breakthrough came at the end of the twentieth century. During this time, Mary Jane West-Eberhard published two seminal works: a review paper (WestEberhard 1989) and a highly influential book (West-Eberhard 2003). In these (and other) publications, she argued that phenotypic plasticity can promote adaptation, novelty, and both intra- and inter-specific diversity. The ensuing decades witnessed the emergence of what has become known as the plasticity-led evolution hypothesis (sometimes called 'PLE'; sensu Levis and Pfennig 2019).

According to the plasticity-led evolution hypothesis, adaptive evolution generally and the evolution of novelty and diversity specifically are set in motion-and potentially directed-by phenotypic plasticity. This view differs from the traditional view in which adaptive phenotypic evolution is triggered by a change in the genome (Figure 9.2a) and where the sole role of environmental change is to alter patterns of selection. With plasticity-led evolution, adaptive phenotypic evolution is triggered by a change in the environment (Figure 9.2b) that simultaneously alters patterns of selection while generating novel developmental variants upon which selection can act.

More precisely, the process of plasticity-led evolution starts when a genetically diverse population experiences a new environment. Because natural environments change constantly, and because these changes are often harmful to the individual (it reduces the match between its phenotype and its environment), organisms have evolved plasticity to produce phenotypes that are better suited for any new conditions they might encounter. In most natural populations, however, different genotypes vary 
(a) Mutation -led evolution

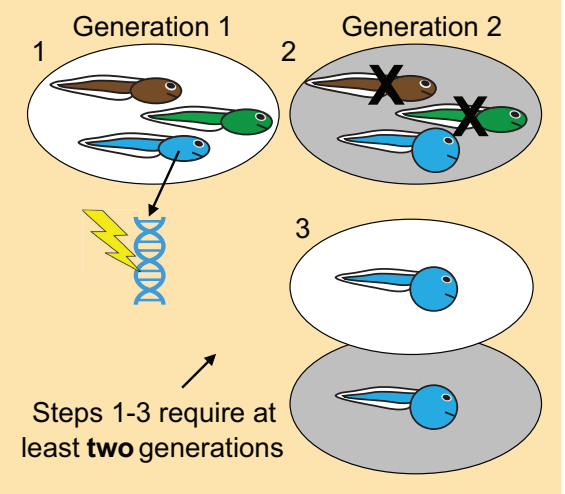

(b) Plasticity -led evolution

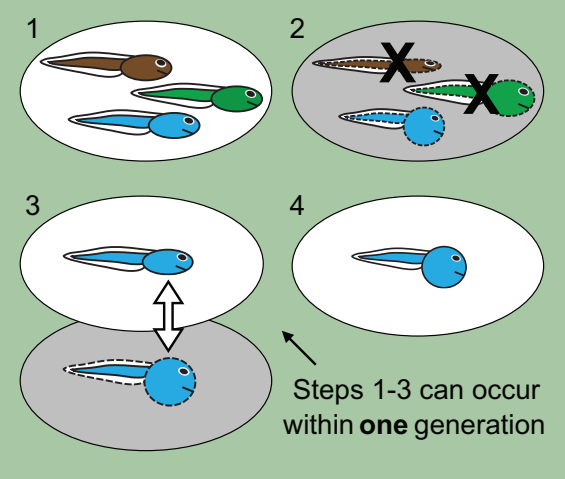

FIGURE 9.2 Alternative routes to novelty. (a) With mutation-led evolution, phenotypes change following a change in the genome. (Step a1) This process begins with a genetic mutation (here, different colors signify different genotypes). (Step a2) Assuming this mutation is not lethal, in the next generation it might cause any individual that inherits it to express a new phenotype (e.g., a 'bulgy' body tadpole form). Moreover, if the population experiences a new environment (indicated by the shaded background), where this form is now favored, selection can disfavor those genotypes that produce poorly adapted phenotypes for the current environment (indicated by an ' $\mathrm{X}$ '). (Step a3) Over evolutionary time, such selection might result in a novel phenotype that is produced regardless of the environment (indicated by different shading). (b) With plasticity-led evolution, phenotypes change following a change in the environment. (Step b1) This process begins when a genetically diverse population (once again, different colors signify different genotypes) (Step b2) experiences a change in its environment (once again, indicated by shading), which induces novel phenotypes (dashed lines). However, different genotypes typically differ in how they respond to an environmental change (indicated by different shapes). Selection can therefore act on such formerly 'cryptic genetic variation' and disfavor those genotypes that produce poorly adapted phenotypes for the current environment (indicated by an ' $\mathrm{X}$ '). (Step b3) If, depending on their environment, individuals produce either this novel phenotype or the ancestral phenotype, then the result is a novel 'polyphenism.' (Step b4) Over evolutionary time, selection might even favor the loss of plasticity (i.e., 'genetic assimilation'), resulting in a novel phenotype that is produced regardless of the environment (indicated by the loss of dashed lines).

in whether and how they respond to any given environmental change (Schlichting and Pigliucci 1998). Once this previously cryptic genetic variation is uncovered by plasticity, selection can remove from the population those phenotypes (and associated genotypes) that are poorly suited for the new environment. If the new environment persists, selection might promote the evolution of quantitative genetic changes that stabilize, refine, and/or extend those phenotypes that are best suited to the new environment. Since environmentally triggered traits are always associated with the environment that triggered their development, they are likely to experience consistent selection and directional modification. This association allows new environments to immediately produce and select among new phenotypes to rapidly refine their expression. 
The refinement process can cause a change in the regulation of plasticity and also in the form of the phenotype, ultimately leading to a better match between phenotype and environment. In the end, plasticity-led evolution results in a new phenotype that was not present in the ancestral population, at least not in a well-adapted form. Thus, plasticity-led evolution can be defined formally as the evolutionary process that occurs when a change in the environment triggers a change in phenotype via phenotypic plasticity, and following the uncovering of cryptic heritable variation in how different individuals respond to this environmental change, the environmentally induced phenotype is refined by selection into a new adaptive form. In other words, plasticity-led evolution is adaptive evolution initiated by an environmentally induced change in a phenotype (plasticity).

The plasticity-led evolution hypothesis explicitly affirms that adaptive phenotypic evolution is not solely a consequence of selection acting on genetic variation. Instead, it highlights that adaptive evolution results from selection acting on heritable developmental variation. This developmental variation, of course, arises as a consequence of both gene products and environmental inputs. Moreover, by placing a major focus on the initial development of new phenotypes, the plasticity-led evolution hypothesis re-orders the sequence of events leading to adaptive evolution (see Section 9.4). Although the plasticity-led evolution hypothesis recognizes genetic variation as a necessary pre-requisite for evolutionary innovation and diversification, it emphasizes how such variation might only be revealed to selection when a change in the environment induces a change in phenotype through plasticity. In doing so, a plasticity-led evolution perspective shifts the focus away from viewing plasticity's role in evolution as a constraining force (sensu Huey et al. 2003) to a constructive one.

But how does plasticity generally, and plasticity-led evolution specifically, promote innovation and diversification? We turn to this topic next.

\subsection{HOW PLASTICITY-LED EVOLUTION PROMOTES INNOVATION AND DIVERSIFICATION}

Phenotypic plasticity — as mediated by plasticity-led evolution-can have far-reaching impacts on the origins of diversity, from promoting diversity and novelty within populations to promoting diversity between species. To discuss these impacts, we will examine plasticity-led evolution's contributions to evolutionary innovation and diversification separately.

\subsubsection{Plasticity-Led Evolution and Innovation}

When discussing the origins of major new organismal features (i.e., evolutionary innovation), it is generally assumed that new traits require genetic changes (Carroll 2008). Indeed, many new features can be traced to genetic changes, including single-gene mutations (e.g., Hoekstra et al. 2006), duplications of large regions of the genome (e.g., Conant and Wolfe 2008), alterations in regulatory sequence (e.g., Chan et al. 2010), and/or a variety of other changes in DNA sequence or content. 
Yet, as mentioned above, biologists have long questioned whether novel features arise solely from genetic changes (West-Eberhard 1989, 2003; Moczek et al. 2011). According to this alternative view, phenotypic plasticity is an important instigator of evolutionary innovation, and plasticity-led evolution should therefore be considered a valid mechanism for generating evolutionary innovation. Here, we discuss three non-mutually exclusive pathways by which plasticity can foster evolutionary innovation: (1) by uncovering cryptic heritable variation; (2) by providing rich targets on which selection can act; and (3) by using exploratory developmental processes.

First, as highlighted earlier in Section 9.2, plasticity can initiate novelty by uncovering cryptic heritable variation (typically, such variation is genetic, but it might also be epigenetic; see Bonduriansky and Day 2018). Cryptic variation is not observed under normal conditions but has observable phenotypic effects under novel (or stressful) conditions (e.g., Gibson and Dworkin 2004). The uncovering of this cryptic variation is crucial for fueling plasticity-led evolution because selection operates on phenotypes, not genotypes, but it can only act on those phenotypes that are actually expressed. This cryptic variation arises as a result of dominance, epistasis, or gene-by-environment interactions/conditional trait expression (Paaby and Rockman 2014). The latter, conditional trait expression (where only a fraction of individuals in a population express a particular trait) is most relevant to plasticity-led evolution. Conditional trait expression often arises when individuals change their phenotype in direct response to different environments (West-Eberhard 2003; i.e., when they express plasticity; Van Dyken and Wade 2010). A common view is that when alternative traits are produced through plasticity, each such trait involves either different genes being expressed or the same gene(s) being expressed at different levels (Snell-Rood et al. 2010). Since phenotypes (and their corresponding genes) are only exposed to selection when expressed, genetic variation accumulates in genes that underpin any phenotype that is not expressed frequently (Paaby and Rockman 2014). Following a change in the environment, this formerly cryptic genetic variation can be uncovered (e.g., by perturbation of silencing/buffering mechanisms; Rutherford and Lindquist 1998), resulting in a novel phenotype. If this environmental change is novel (i.e., it has not been previously experienced by the focal lineage), then many of these new phenotypic variants will likely be maladaptive. However, some such variants will likely be adaptive in the novel environment just by chance (West-Eberhard 2003). In contrast, if the new environment is not novel, then some phenotypic variants will likely be biased toward being adaptive in the altered environment (Parsons et al. 2020). Whether adaptive or not, environmentally mediated changes to development (i.e., plasticity) generate a pool of heritable phenotypic variation on which selection can act. Such selection can then further refine those variants that happen to be well-suited to the new environment.

Second, plasticity can also foster evolutionary innovation by providing rich targets for diversifying selection. In particular, because plasticity typically results from a complex interaction between both gene products and environmental inputs-i.e., it involves a gene-by-environment interaction-it provides numerous targets on which selection can act to promote novelty. Indeed, from recent studies into the developmental underpinnings of complex traits (reviewed in Glazier et al. 2002; Wu and Lin 2006), it is clear that many phenotypic outcomes are the result of multifaceted 
regulatory interactions during development. It is also clear that evolutionary mechanisms operating on these regulatory interactions can lead to novel traits (Carroll 2008; Moczek et al. 2011; Levis and Pfennig 2020). Yet, while the complexity of developmental systems is relevant to the evolution of any novel trait, it may be particularly important in the context of plasticity because of additional interactions with the environment. The number and diversity of interactions among regulatory factors on the one hand (such as hormones, receptors, transcription factors) and environmental conditions on the other provide numerous opportunities for development to be perturbed and new phenotypic variants to arise (e.g., from changes in the timing, location, or strength of interactions). Consider, for example, that many signaling molecules and signal transducers can modify, inhibit, or promote (i.e., regulate) activities performed by other molecules, and that these regulatory molecules typically have numerous targets (e.g., Payne et al. 2014). This diversity and abundance of targets may increase the probability of a trait becoming decoupled from its environmental cue, establishing new connections with an environmental cue, and/or experiencing various other modifications to its expression (Ehrenreich and Pfennig 2016).

Moreover, because development tends to be 'modular' - meaning that it typically entails multiple sets of interacting parts or 'modules' that are relatively autonomous with respect to each other (Schlosser and Wagner 2004)_ample opportunity exists for regulatory networks to evolve (semi-)independently of each other. Such semiindependent evolution enables some modules (and their associated phenotypes) to develop greater elaboration and refinement with minimal (or no) effects on other modules in the same organism (West-Eberhard 2003; Snell-Rood et al. 2010; e.g., the fore and hindwings of a butterfly or the different types of teeth in a mammal). This means that selection can simultaneously refine multiple plastic phenotypes: each phenotype is only subject to selection when it is expressed, and its refinement may have little impact on other phenotypes (Levis and Pfennig 2019). Thus, network complexity (e.g., number and types of regulatory connections), in conjunction with semi-independent evolution afforded by modular development, provides ample opportunity for the generation of novel phenotypes that can subsequently be refined by selection into adaptive traits.

Finally, plasticity can contribute to novelty through exploratory mechanisms (Levis and Pfennig 2020). These mechanisms typically operate through a process of developmental selection in which some form of environmental sampling is followed by developmental reinforcement of those responses that are most beneficial (Snell-Rood et al. 2018). The 'environment' being sampled may be internal (e.g., cytoskeleton formation) or external (e.g., an animal sampling various foods). Such mechanisms include but are not limited to (Kirschner and Gerhart 1998): cytoskeleton formation, neuron growth and development, plant stem and root growth, vertebrate adaptive immunity, habitat choice, and trial-and-error learning. In general, exploratory mechanisms can be highly sensitive to local conditions and thereby produce adaptive developmental outcomes even in novel environments (Snell-Rood 2012).

Exploratory mechanisms are a powerful force during plasticity-led evolution. Because they use fine-grained local responses generated by subunits of the larger phenotype, exploratory mechanisms can yield phenotypes that are well-suited to current 
conditions (Kirschner 1992; Snell-Rood 2012; Snell-Rood et al. 2018; Ducatez et al. 2020). Indeed, exploratory mechanisms can produce appropriate phenotypes even under novel conditions (Lande 2014). Subsequently, the high costs typically associated with exploratory mechanisms may make the phenotypes they produce more likely to be fixed by selection (Scheiner et al. 2017; Snell-Rood et al. 2018) so that any burden of maintaining plasticity can be lessened.

To illustrate how exploratory mechanisms can generate novelty, recall from above that behavior, in particular, has long been recognized as playing a key role during plasticity-led evolution (e.g., Baldwin 1896; Wcislo 1989; Bateson 2004; Duckworth 2009; Lister 2014). Indeed, behavior is often described as 'hyperplastic.' This is because of the wide array of behavioral changes that many organisms can exhibit depend on environmental context. (As an aside, even organisms lacking brains can assess and respond to different environmental conditions. For example, bacteria and plants are known to move consistently toward or away from specific environmental stimuli.) While behavior may play a leading role in generating new selective pressures that foster evolutionary innovation (see Section 9.2), behavior (or any exploratory mechanism for that matter) may also be important for reducing the amount of environmental variation that an individual experiences. For example, a reptile behaviorally shifting to a shaded habitat during the hottest time of day reduces the temperature variation it experiences, and consequently, influences how selection acts on heat tolerance physiology (Huey et al. 2003). By narrowing the range of environments an organism experiences, behavior can help drive specialization to a particular environment. Specialization can, in turn, channel selection to favor other aspects of the phenotype (e.g., physiological or morphological) that work well in the new environmental conditions.

Ultimately, such specialization wrought by plasticity-led evolution might lead to a polyphenism (Figure 9.1). Indeed, the alternative phenotypes that characterize many polyphenisms are each tightly coupled to specific environmental conditions (Nijhout 2003). Although the evolutionary importance of these phenotypic alternatives has been underestimated, the evolution of a polyphenism may have important implications for the origins of major new features. This is because many (if not all) novel features likely first appeared evolutionarily as an alternative phenotype within a population (West-Eberhard 2003). Thus, as first suggested by Weismann (1882), the alternative phenotypes that constitute polyphenism might be an important phase in the evolution of major adaptive novelties that characterize different species and higher taxa.

In short, plasticity-led evolution may play a key role in evolutionary innovation at all levels of biological organization.

\subsubsection{Plasticity-Led Evolution and Diversification}

Phenotypic plasticity can contribute to diversification by promoting intra- and inter-specific divergence, speciation, and adaptive radiation (West-Eberhard 2003; Schlichting 2004; Pfennig et al. 2010). Below, we discuss how plasticity-led evolution promotes diversification at each level separately. 
First, plasticity-led evolution contributes to intraspecific diversification by fostering the evolution of novel phenotypes. Once new phenotypes are produced through plasticity, plasticity-led evolution can result in their further elaboration. Specifically, when plasticity is favored, plasticity-led evolution can promote polyphenism, thereby generating pronounced diversity within species (Figure 9.1). Conversely, when plasticity is not favored (as when its costs exceed its benefits or when reliable cues for adaptive plasticity are absent; see Berrigan and Scheiner 2004), then plasticity-led evolution can result in a constitutively expressed (i.e., 'fixed') trait evolving from a previously environmentally induced trait. If different populations of the same species fix alternative forms for the trait, and especially if these populations differentially elaborate and refine these forms, then intraspecific diversity has increased. The loss of plasticity might occur for two reasons (Pigliucci et al. 2006; Ehrenreich and Pfennig 2016). First, when plasticity is costly (Murren et al. 2015), selection can actively eliminate it, leading to fixation of the favored phenotype (i.e., genetic assimilation occurs; sensu Waddington 1953; see also Scheiner and Levis 2021 in this volume). Second, plasticity can be lost through mutational degradation or genetic drift (Masel et al. 2007), as might occur when non-favored phenotypes are seldom expressed and thereby experience relaxed selection (Van Dyken and Wade 2010). However, regardless of whether plasticity-led evolution results in a new genetically fixed trait or a polyphenism, it can enhance intraspecific diversity.

Plasticity-led evolution can also facilitate divergence between species. Consider this passage from E. O. Wilson (1992, p. 174):

Imagine a case in which two such species have been squeezed together in the same communities long enough for evolution to occur. When they first came into contact, they were elastic and could diverge in their habits enough to lessen competition. The differences were phenotypic, the result of environment and not genes. The compression occurred in traits that were relatively easy to change, most likely by a retreat from parts of the habitat and diet by one or both of the species. As the generations passed, genetic differences arose and hardened the distinction between the two species.

Wilson is proposing a scenario in which adaptive differences between competing species are initially mediated by plasticity. Later, these differences become fixed ('hardened'). In essence, he is describing plasticity-led 'character displacement'; that is, trait evolution that arises as an adaptive response to competition between species (Pfennig and Pfennig 2012). In a number of taxa, such competitively induced plasticity appears to have preceded the evolution of fixed genetic differences between populations in sympatry with a heterospecific competitor versus populations in allopatry (reviewed in Pfennig and Pfennig 2012, pp. 98-102). Because plasticity can generate rapid, widespread, and adaptive changes in resource-use traits, it might play a general and important role in character displacement specifically and population divergence generally.

Essentially, plasticity promotes diversification because it facilitates a population's ability to cross selective valleys and thereby access nearby selective peaks (Price et al. 2003; Schlichting 2004; selective 'valleys' and 'peaks' refer to phenotypes for which the population mean fitness is at a minimum and maximum, respectively). To reach such a peak through the traditional process involving accumulation of small genetic 
(a)

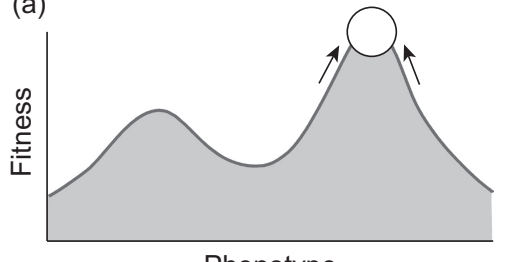

Phenotype

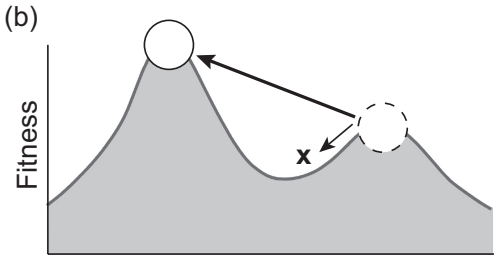

Phenotype

FIGURE 9.3 How plasticity promotes diversification by facilitating 'valley crossing.' (a) A well-adapted population should evolve to produce a mean phenotype that is associated with highest fitness on the adaptive landscape (the 'fitness maximum,' the position of which is indicated here by a circle). (b) However, when the environment changes, the adaptive landscape is likely to change also, such that the population mean phenotype is no longer at the new fitness maximum. This new fitness maximum would be inaccessible through incremental evolution because of an intervening fitness valley; selection should generally not take a population through such a valley. However, in a population that has evolved plasticity (particularly polyphenism; see Figure 9.1), the individual members of the population could cross this fitness valley in 'developmental time' by facultatively expressing an alternative phenotype that is associated with higher fitness; including one at the new fitness maximum.

changes, a population must first cross a fitness valley (i.e., it must produce offspring with phenotypes that are associated with lower fitness than the parents). Such valley crossing via the evolution of small genetic changes is normally prevented by selection. However, with plasticity (and especially with polyphenism), valley crossing unfolds in developmental time rather than in evolutionary time (Figure 9.3). By facultatively expressing an alternative phenotype closer to the fitness optimum, a population can traverse a valley rapidly_potentially in one generation. Consequently, in a population experiencing rapidly changing environmental conditions, plasticity enables populations to explore the fitness landscape (initially, through development; potentially via exploratory mechanisms described in Section 9.3.1), increasing its chances of diversifying.

Moreover, because plasticity can promote population divergence, and because the same factors that promote population divergence should also foster speciation (Schluter 2000), plasticity may thereby facilitate speciation (Pfennig et al. 2010). For example, there is evidence that matings between populations that differ in the expression of environmentally induced alternative phenotypes result in offspring of low fitness (Pfennig and Rice 2007). This reduced fitness could favor reproductive isolation between such populations and thereby possibly lead to speciation. Indeed, speciation may proceed especially rapidly when alternative phenotypes are environmentally induced. This is because a sudden change in the environment can simultaneously induce and favor a single alternative phenotype. Furthermore, any population differences that initially arose through plasticity might undergo genetic assimilation. This process might thereby contribute to the rapid accumulation of genetic differences between populations that, in turn, enhance reproductive isolation. Consistent with these arguments, there is growing support for the hypothesis that plasticity—particularly polyphenism—contributes to speciation (Meyer 1993; Smith 
and Skúlason 1996; Pfennig and McGee 2010; Pfennig et al. 2010; Hendry 2016). For example, clades in which resource polyphenism has evolved are more species rich than sister clades (Pfennig and McGee 2010). However, since polyphenism is a potential outcome of plasticity-led evolution, plasticity-led evolution's main contribution to speciation may be facilitating the evolution of refined plastic responses that can subsequently generate the conditions favoring reproductive isolation and speciation.

Lastly, plasticity-led evolution might promote adaptive radiation (West-Eberhard 2003; Wund et al. 2008; Schneider and Meyer 2017), which occurs when the members of a single phylogenetic lineage diversify rapidly into numerous descendent lineages that occupy a wide variety of ecological niches (Simpson 1953b; Schluter 2000). Adaptive radiation mediated by plasticity-led evolution is sometimes referred to as the 'flexible stem hypothesis' (West-Eberhard 2003). Under this hypothesis: (1) the diverse forms produced by an adaptive radiation started out as environmentally induced forms; and (2) the nature of this ancestral plasticity can influence the nature of the radiation (West-Eberhard 2003; p. 565). This model further predicts that ancestral, plastic responses within a species should reflect the fixed trait differences observed between closely related species within the same clade (Gomez-Mestre and Buchholz 2006; Wund et al. 2008; Gibert 2017). This mirroring occurs because of divergent selection acting on the alternative phenotypes induced by plasticity and ultimately driving fixation of the alternative phenotypes in different lineages.

This model can help explain a characteristic of many adaptive radiations: 'parallelism,' in which similar traits have been repeatedly derived from the same ancestral population or phenotypic form (the resulting pattern is sometimes dubbed 'replicate radiation'; Schluter and Nagel 1995; Losos et al. 1998). A plasticity-led evolution framework emphasizes that the environment not only exerts parallel selective pressures but also generates parallel distributions of traits - owing to parallel induction of ancestral plastic responses - which, together, can lead to highly deterministic outcomes (Parsons et al. 2020). Thus, plasticity-led evolution may not only promote biodiversity but also govern the shape that biodiversity takes.

\subsection{EVALUATING PLASTICITY-LED EVOLUTION}

Obviously, one can test the plasticity-led evolution hypothesis experimentally by evolving populations under controlled laboratory conditions; that is, using an 'experimental evolution' approach (Garland and Rose 2009). A major benefit of experimental evolution is that it can be designed to test specific hypotheses. This approach was employed by Waddington in his classic studies of genetic assimilation (Waddington 1942, 1952, 1953). Ideally, one would choose to study subjects that can also be assayed in the wild in order to corroborate laboratory findings (e.g., see Suzuki and Nijhout 2006). Nevertheless, experimental evolution has limitations. First, it can only be used with organisms that have short generation times (e.g., bacteria, yeast, fruit flies). Second, some evolutionary processes might be too slow to observe within the span of a research grant or even a researcher's lifetime (a case in point is the limited insights that experimental evolution has provided into speciation). Finally, one is always left with the uncertainty of whether evolution in the laboratory reflects how it actually unfolds in natural populations. 
Using natural populations to evaluate the origins of extant traits (or taxa) is challenging because only the final products of the evolutionary process are likely to still exist. In these cases, the main difficulty is that once a trait has evolved, its evolution cannot be studied in situ. To circumvent this problem, two general approaches could be employed.

First, phylogenetic methods (e.g., ancestral state reconstruction) could provide information on the timing of trait evolution and identify the order of shifts in trait regulation from environmental induction to genetic control (and vice versa) among lineages (Schwander and Leimar 2011; Schlichting and Wund 2014). In this way, one could determine if a trait has shifted from being plastic to being genetically fixed in some lineages that are phylogenetically embedded in a larger clade where plasticity is retained. This approach requires a well-resolved phylogeny and understanding of the degree of plasticity in those taxa included in the phylogeny.

A second widely used approach (Schlichting and Wund 2014; Levis and Pfennig 2016) is to compare the reaction norms (i.e., the trait's plasticity as estimated from the shape or slope of the line between trait values in alternative environments) of two different types of lineages: one that possesses the focal, potentially canalized, trait (representing the 'derived' condition) and one that is closely related to the former lineage but that lacks the focal trait (representing the 'ancestral' condition and that can therefore serve as an 'ancestor-proxy'). For example, one could study natural populations that have undergone a range expansion (Badyaev et al. 2002). In such cases, one could compare individuals from the 'sink' population (representing the derived condition) to those in the 'source' population (representing the ancestral condition). Reaction norms of these two types of lineages (or across a group of lineages ranging from 'more ancestral' to 'more derived') can be compared when both lineages are reared in both the derived environment (i.e., the environment in which the novel trait is associated) and the ancestral environment. Such a comparison can help infer what the common ancestor of the groups might have looked like in terms of its trait values and plasticity and how these features were elaborated in the focal lineage. Using this approach, one could then test the critical predictions of plasticity-led evolution (Table 9.1; Levis and Pfennig 2016). Indeed, in recent years, such methods have been used to evaluate plasticity-led evolution in a wide variety of taxa and traits (Table 9.2).

Finally, if plasticity-led evolution is being implicated in speciation and/or adaptive radiation, then there should be evidence that: (1) reproductive isolation evolved after lineage divergence, and (2) lineage divergence occurred (at least in part) because of phenotypic divergence via plasticity-led evolution (i.e., predictions 1-4 in Table 9.1 have support). Ideally, one should also show that interspecific (fixed) differences mirror intraspecific (environmentally induced) differences (Gomez-Mestre and Buchholz 2006; Wund et al. 2008).

\subsection{PLASTICITY-LED EVOLUTION: CONSENSUS, CONTROVERSY, AND CHALLENGES}

In all fields of research, it is important to take stock of where the field has been, where it stands presently, and where the field needs to go to continue to make progress. Here, we highlight areas of consensus surrounding plasticity's role in evolution before 


\section{TABLE 9.1}

\section{Key Predictions of the Plasticity-Led Evolution Hypothesis}

\section{Prediction}

1. The focal trait can be environmentally induced in lineages showing the ancestral state

2. The focal trait exhibits evidence that heritable variation (i.e., variation upon which selection could act to refine the trait) has been uncovered by the novel conditions

3. The focal trait has undergone an evolutionary change in its degree of plasticity and/or form in lineages with the derived trait

4. The focal trait has experienced adaptive refinement as it is repeatedly induced and exposed to selection

\section{Explanation}

The fundamental prediction of plasticity-led evolution is that the novel trait should exhibit ancestral plasticity. In other words, the derived trait (or components thereof) should be environmentally induced in lineages that normally lack expression of the trait, but only when they experience the derived environment (West-Eberhard 2003). This would suggest that the common ancestor between the ancestral and derived lineage(s) likely possessed such plasticity as well.

Confirmation of heritable variation is necessary to help eliminate the possibility that the novel trait arose solely through lineage-specific mutations (as in Fanti et al. 2017; Santos et al. 2017), and instead, is the product of ancestral developmental plasticity. Such evidence could be inferred if the trait (or its components) have higher additive genetic variance/heritability when ancestral lineages are reared under derived conditions (Gibson and Dworkin 2004; Ledón-Rettig et al. 2014; Noble et al. 2019).

Evolutionary change in plasticity should manifest as changes to the slope, curvature, and/or elevation of the reaction norm (Crispo 2007). Finding that selection has led to the complete loss of plasticity would imply that the trait has undergone genetic assimilation. Whereas finding that plasticity has increased might point toward the possible evolution of a polyphenism. Either outcome (or something in between) could be expected depending on the rate of environmental change, the strength of selection acting on plasticity, and/or the phenotypes induced by plasticity.

The focal trait should exhibit a pattern of frequency-dependent adaptation (sensu Levis and Pfennig 2019) such that the more frequently it is induced, the greater (and potentially more rapid) refinement it should experience. Finding such a difference is likely when using distinct species for ancestor-derived comparisons. Intraspecific (e.g., among population) comparisons can help corroborate that this same process has operated at both the microevolutionary and macroevolutionary levels.

discussing some of the reasons why the topic remains controversial. We then devote most of this section to addressing two key challenges to plasticity-led evolution.

\subsubsection{Plasticity-Led Evolution: Where Is the Consensus?}

Although the notion that plasticity can impact evolution is not new (see Section 9.2; see also Futuyma 2021; Pfennig 2021 in this volume), the field has undergone a renaissance over the past two decades (as evidenced by a dramatic uptick in publications; see Forsman 2014). Here, we call attention to three areas of consensus between plasticity researchers and evolutionary scientists. 


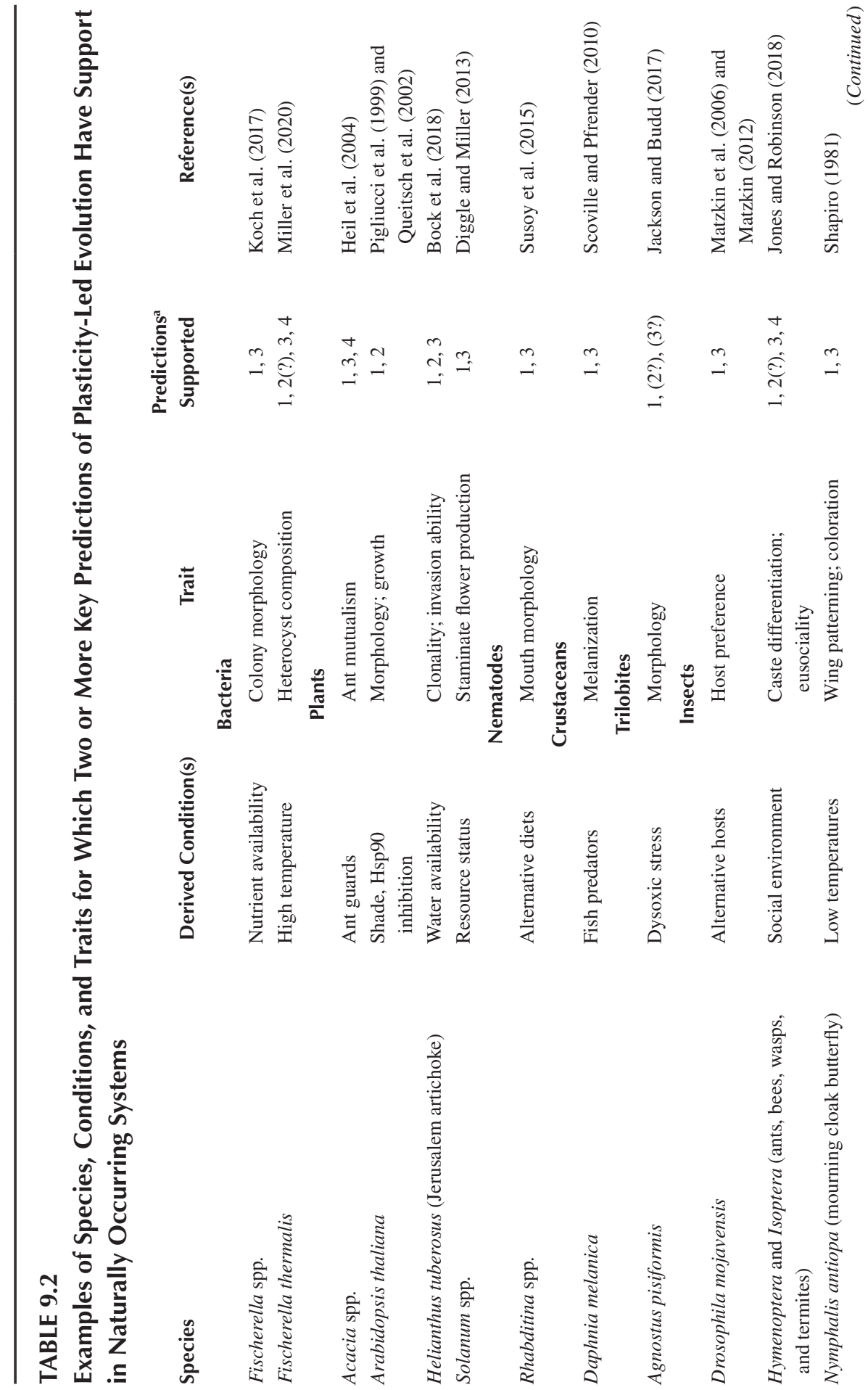




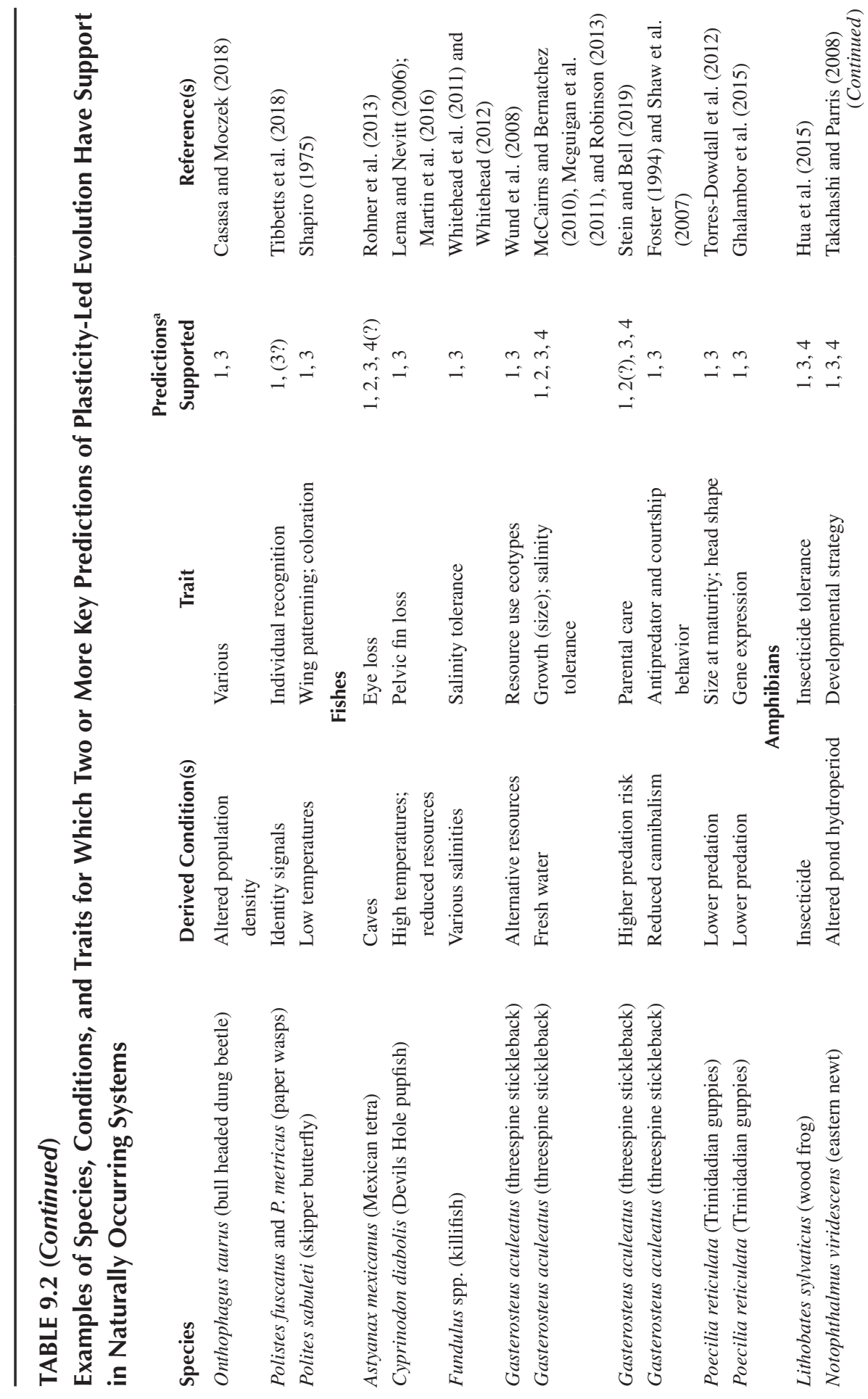




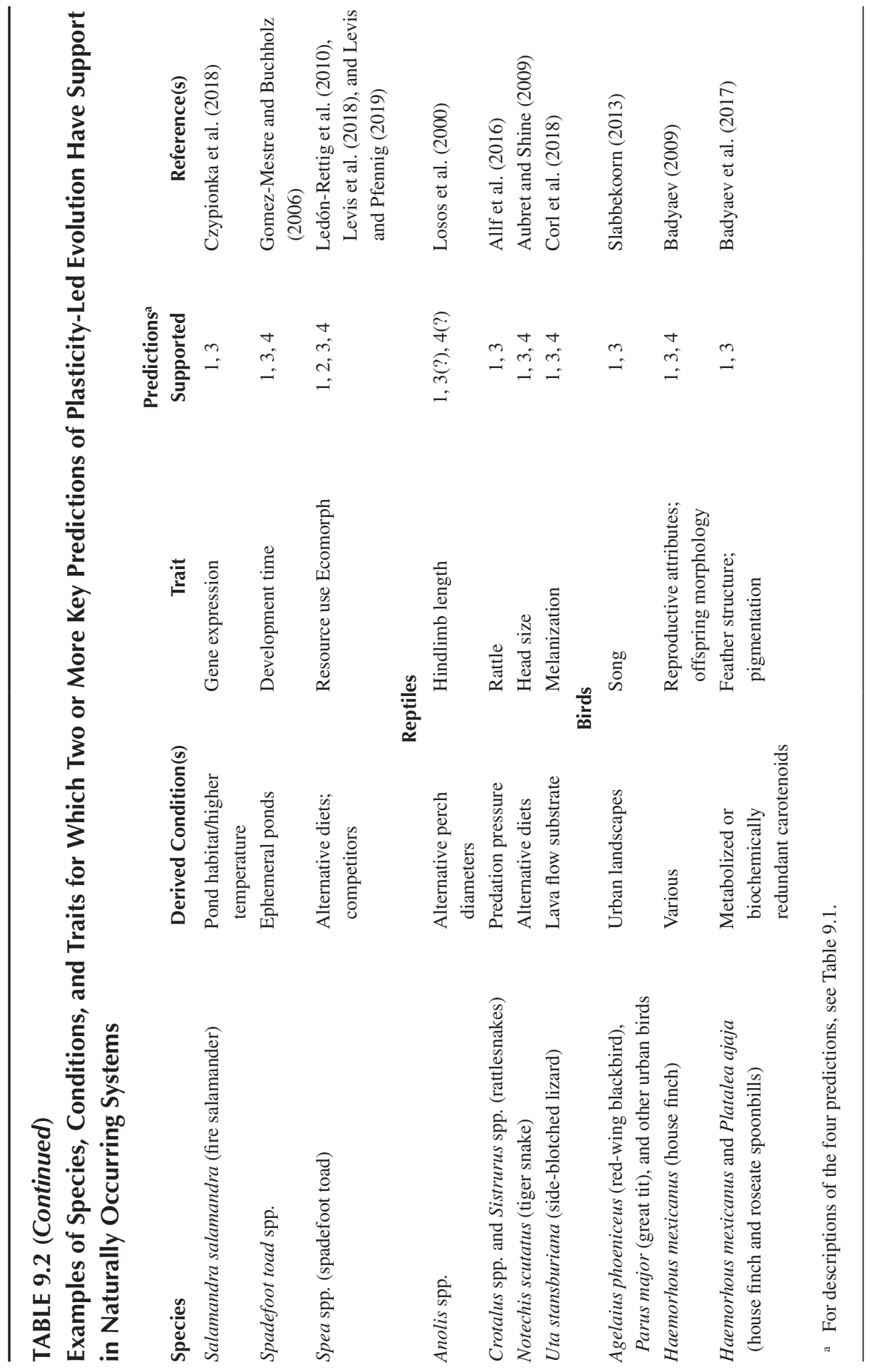


First, a quick review of the literature reveals that most evolutionary biologists appreciate that plasticity is often beneficial. Indeed, most scientists seem to recognize that plasticity provides an important mechanism for organisms to deal adaptively with the stress accompanying changing environments (Losos 2014). Second, precisely because of this first point, most evolutionary biologists also appear to appreciate that plasticity can 'buffer' organisms against environmental change (Fox et al. 2019) and thereby enable populations to persist in the face of novel or changing environments (Ducatez et al. 2020; see also Diamond and Martin 2021; Pfennig 2021 in this volume). Such persistence is important because it allows lineages to 'buy time' until they can evolve further (Pennisi 2018). Finally, most evolutionary biologists acknowledge that plasticity can readily evolve, as long as there is genetic variation in the response. Appreciation of these three facts is critical, for they form the bases for plasticity-led evolution.

\section{BOX 9.1 ANSWERS TO NINE COMMONLY ASKED QUESTIONS ABOUT PLASTICITY-LED EVOLUTION}

1. What is plasticity-led evolution? Plasticity-led evolution is the evolutionary process that occurs when a change in the environment triggers a change in phenotype via phenotypic plasticity, and following the uncovering of cryptic heritable variation in how different individuals respond to this environmental change, the environmentally induced phenotype is refined by selection into a new adaptive form.

2. Isn't plasticity-led evolution just selection promoting evolutionary change by acting on quantitative genetic variation? Yes, but under plasticity-led evolution, a change in the environment exposes that quantitative genetic variation to selection (via plasticity) in the first place.

3. Is the basic idea behind plasticity-led evolution really new? Not really. As we highlighted in Section 9.2, the rudiments of the idea go back over a century. However, the modern framing of the hypothesis-with its emphasis on environmental induction and uncovering of previously cryptic genetic variation-is relatively new.

4. If genetic variation must be present before plasticity-led evolution can occur, how can one say that genes are 'followers?' Because genes are necessary for producing phenotypes, they obviously cannot be 'followers' in the strictest sense. The 'genes as followers' perspective emphasizes that environmental induction and uncovering of previously cryptic genetic variation may have been the trigger that instigated many examples of phenotypic evolution. This viewpoint differs from a strict mutation-led evolution viewpoint, in which genes can be more clearly described as 'leaders.'

5. Isn't plasticity-led evolution another way of saying 'plasticity evolves,' which we already knew about? What's new? Plasticity-led 
evolution is not synonymous with 'plasticity evolves.' Plasticity-led evolution does involve the evolution of plasticity (see Figure 9.2b), which researchers have indeed long known about. What is new is that the evolution of plasticity can have important ramifications: in particular, it might set in motion a sequence of events leading to the origin of new traits and even new species, as we describe in this chapter.

6. Doesn't plasticity-led evolution merely describe the evolution of incomplete penetrance? No. In genetics, 'penetrance' refers to the proportion of individuals carrying a particular allele that express the trait normally associated with that allele. An allele exhibits 'incomplete penetrance' if some individuals carrying the allele do not express the trait. Because incomplete penetrance can arise owing to environmental factors, it could be said to be caused by phenotypic plasticity. However, not all cases of incomplete penetrance are due to plasticity; incomplete penetrance can also be caused by other factors, such as genetic modifiers. Moreover, as noted in our answer to the previous question, plasticity-led evolution entails more than just the evolution of plasticity.

7. Is plasticity-led evolution the only way that plasticity can impact evolution? No, it is not. In fact, in some cases, plasticity might actually impede evolution and in other cases have no impact at all. Moreover, plasticity-led evolution is not the only way that plasticity can facilitate evolution (see also Pfennig 2021 in this volume).

8. Does plasticity-led evolution contradict existing evolutionary theory? Not at all. As with every case of adaptive evolution, plasticityled evolution entails selection acting on underlying heritable (e.g., genetic) variation (see also Futuyma 2021; Pfennig 2021; Schlichting 2021 in this volume). However, plasticity-led evolution does place greater focus on the proximate mechanisms by which phenotypic variation arises and on the evolution of development.

9. Are there any examples of a trait that has arisen via plasticityled evolution? There are numerous possible examples, including many from natural populations (see Table 9.2). However, without a time machine, one can never be certain that a particular trait arose via plasticity-led evolution rather than via mutation-led evolution; of course, the converse is also true!

\subsubsection{Plasticity-Led Evolution: Where Is the Controversy?}

Where consensus over plasticity's role in evolution starts to break down is when talking about whether plasticity can promote evolution directly, i.e., via plasticityled evolution. One problem here is that the rationale behind plasticity-led evolution has generally not been articulated clearly. This lack of clarity has fostered confusion over what is meant by plasticity-led evolution (Wray et al. 2014), when, in fact, plasticity-led evolution fits comfortably within modern evolutionary theory (see Box 9.1; see also Futuyma 2021; Pfennig 2021; Schlichting 2021; and WestEberhard 2021 in this volume). An unfortunate consequence of this confusion is 
that many textbook authors (whether intentionally or not) have simply ignored the topic altogether. For example, very few college-level evolution textbooks even list the terms 'phenotypic plasticity' and 'genetic assimilation' in their indices [for an exception, see Futuyma and Kirkpatrick's (2017) textbook, in which they discuss plasticity-led evolution on pp. 394-395]. Genetic assimilation, in particular, has long been controversial (Simpson 1953a; Williams 1966; Orr 1999; de Jong 2005; Wray et al. 2014; see also Scheiner and Levis 2021 in this volume). Thus, because students are not being exposed to the plasticity-led evolution hypothesis (and related ideas), ignorance of the topic persists.

However, perhaps the main impediment to a greater appreciation of plasticityled evolution is the perceived lack of empirical evidence in support of it (Levis and Pfennig 2016). We say "perceived lack" because empirical support for plasticity-led evolution has increased dramatically in recent years (e.g., see Table 9.2). Nevertheless, because "the best way to elevate the prominence of genuinely interesting phenomena such as phenotypic plasticity ... is to strengthen the evidence for their importance" (Wray et al. 2014, p. 164), in the long run, devising and implementing rigorous tests remains essential to widespread acceptance of plasticity-led evolution.

\subsubsection{Plasticity-Led Evolution: Where Are the Challenges?}

We now address two clear challenges to the plasticity-led evolution hypothesis. As we describe, these challenges focus on whether or not a plasticity-led evolution interpretation is necessary and/or sufficient to explain evolutionary innovation and diversification.

One key challenge to the plasticity-led evolution hypothesis is a debate over the order of events versus the importance of those events (e.g., Futuyma 2015). We refer to this debate as being over 'sequence' versus 'significance,' respectively. This debate specifically takes issue with a claim made by Mary Jane West-Eberhard (2003) when discussing plasticity-led evolution in her influential book, Developmental Plasticity and Evolution (pp. 157-158):

Most phenotypic evolution begins with environmentally initiated phenotypic change... Gene-frequency change follows, as a response to the developmental change... Genes are followers, not necessarily leaders, in phenotypic evolution.

Of course, as we have stressed throughout this chapter, pre-existing genetic variation is the starting point for plasticity-led evolution. This means that genes cannot be followers in the strictest sense. Or as Douglas Futuyma (2017, p. 6) has put it:

Genes are 'followers' only to the extent that genetic assimilation... 'fine-tunes' an adaptation that had already evolved by selection and genetic variation.

It is important to note that both the sequence and significance viewpoints emphasize how downstream quantitative genetic changes modify and refine an environmentally induced phenotype. The major difference between these two perspectives is that the sequence viewpoint (as articulated by Futuyma) emphasizes the role of past selection and existing variation, whereas the significance viewpoint (as articulated by West-Eberhard) emphasizes the relative importance of different processes during adaptive evolution: development of novel phenotypic variants versus selection on those variants. Thus, the challenge is that plasticity-led evolution may not constitute a 
significant departure from, or extension of, evolutionary theory: selection and genetic variation precede any plastic responses that ignite a sequence of plasticity-led evolution (see also Futuyma 2021 and West-Eberhard 2021 in this volume). However, even if past selection may have acted and/or genetic variation already exists, the significance view would argue that these factors are not developmentally or phenotypically important until an environmental change makes them so. In this way, the environmental induction and uncovering of previously cryptic variation (regardless of how that variation accumulated) play the major (i.e., leading) role during a particular bout of phenotypic evolution.

The significance viewpoint also elevates the importance of environmental change during the initiation of novelty by giving it equal footing with mutation. Indeed, as we describe below, environmentally induced phenotypic change may have even greater evolutionary potential than a mutation. Whereas novel genotypic variation ultimately stems from new mutations, novel phenotypic variation stems from developmental innovation, which itself depends on both genetic and environmental factors. Therefore, when discussing the origin of novel phenotypes, one must consider the roles of both gene products and environmental inputs. Furthermore, these two inputs are potentially evolutionarily interchangeable, meaning that selection can slide trait regulation anywhere along a continuum from greater environmental control (potentially resulting in a polyphenism) to greater genetic control (potentially resulting in a fixed trait via genetic assimilation). Moreover, because phenotypic variation is required for evolution by natural selection, environmental change is similar to a new mutation in that it can be considered a first-order cause of evolutionary novelty. That is, environmental inputs during development give rise to new phenotypes before natural selection sifts among those phenotypes. Importantly, this view re-orients how we think of environmental change by highlighting its dual role as a generator and then selector of phenotypic variation.

In addition to being partners with gene products during developmental-and thus phenotypic-innovation, environmentally induced phenotypic change may harbor greater evolutionary potential than mutationally induced change for at least three, non-mutually exclusive reasons (West-Eberhard 2003; Levis and Pfennig 2016). First, changes in the environment often affect many individuals simultaneously. This situation is in contrast to a genetic mutation, which initially affects only one individual and its immediate descendants. Such widespread impact of environmental change enables a newly induced trait to be tested among diverse genetic backgrounds, thereby increasing the opportunity for selection to act and for subsequent adaptive refinement to occur.

Second, although the chance that a particular mutation will occur is not influenced by whether or not the organism is in an environment in which that mutation will be advantageous - in other words, adaptively directed mutation does not occur (Sniegowski and Lenski 1995) —environmentally triggered traits are always associated with a particular environment: the one that triggered it. Therefore, environmentally induced traits are more likely than mutationally induced novelties to experience consistent selection and directional modification (West-Eberhard 2003). This constancy allows new environments to immediately produce and select among new phenotypes and rapidly refine their expression (Badyaev 2005). While transmission of environmentally induced novelties across generations requires some mechanism of inheritance, which is most likely genetic, the same is true of mutationally induced 
novelties: both require a pre-existing genetic (or otherwise heritable) background in which to integrate and transmit novel information.

Third, the waiting time for a new mutation to arise and increase in frequency in a population can be prohibitively long (Charlesworth 2020). In contrast, new phenotypic variants triggered by the environment are produced over developmental time, i.e., over a single individual's lifetime. The speed with which environmentally induced change can arise might prove critical in the face of a rapidly changing environment, where a delay in producing adaptive new phenotypic variants might result in a population going extinct (Pfennig and Pfennig 2012; Ducatez et al. 2020).

A second major challenge to plasticity-led evolution is its alleged inability to discriminate among alternative hypotheses (Kovaka 2019; Noble et al. 2019). This problem largely stems from the methodological approaches that have been used to study plasticity-led evolution. Specifically, since most studies focus on characteristics of extant taxa using ancestor-derived comparisons of phenotypic responses, it is difficult (or perhaps, in some cases, impossible) to determine if ancestral plasticity did indeed drive evolutionary change or if a de novo lineage-specific mutation did. That is, without knowing the molecular basis of a plastic response, one cannot determine if a new mutation preceded and drove the development of the derived phenotype or, conversely, if cryptic genetic variation-coupled with environmental change-did.

Notably, the key predictions outlined in Table 9.1 do not address these difficulties. Yet, these concerns are irrelevant if the key predictions are not supported. Thus, while the predictions outlined in Table 9.1 are necessary to demonstrate plasticity-led evolution, they may not be sufficient to rule out alternative hypotheses. We speculate that a discriminatory signature of plasticity-led evolution might combine evidence of: (1) a soft selective sweep (i.e., adaptation from standing genetic variation; Messer and Petrov 2013); (2) conditional expression (Van Dyken and Wade 2010); and (3) phenotypic, developmental, and environmental change (e.g., support for the predictions in Table 9.1). A good example that extends beyond the key predictions described in Table 9.1 comes from a study of craniofacial morphology in cichlids by Parsons et al. (2016). In this study, researchers followed the framework outlined in Section 9.4 for evaluating plasticity-led evolution. However, they went a step further by identifying environmentally sensitive quantitative trait loci and determining that the derived allele of one of these loci exhibited markedly reduced environmental sensitivity than the ancestral allele. That is, they were able to identify a particular regulatory locus whose evolution matches predictions of genetic assimilation via plasticity-led evolution. Recently, more attention has been paid to the evolution of plasticity mechanisms and how various mechanisms might influence plasticity-led evolution in different ways (SnellRood et al. 2018; Levis and Pfennig 2020; see also Goldstein and Ehrenreich 2021; Ledón-Rettig and Ragsdale 2021 in this volume). This focus on mechanisms, coupled with additional theoretical and modeling efforts (e.g., Scheiner 2014; Scheiner et al. 2017), may provide tractable approaches for dealing with the difficulty of discriminating among alternative hypotheses (Kovaka 2019).

\subsection{CONCLUSIONS}

Explaining the origins of diversity is a longstanding problem in evolutionary biology. As we have seen, phenotypic plasticity can have far-reaching impacts on the 
origins of diversity, ranging from promoting diversity and novelty within species (Figure 9.1) to promoting population divergence, speciation, and even adaptive radiation. Accordingly, incorporating plasticity generally and plasticity-led evolution specifically into evolutionary thinking provides a richer understanding of how and why living things diversify. Finally, in Box 9.1, we provide answers to some commonly asked questions about plasticity-led evolution, and in Box 9.2, we provide some suggestions for future research.

\section{ACKNOWLEDGMENTS}

For commenting on earlier drafts of this chapter, we thank Emily Harmon, Andrew Isdaner, Pat Kelly, Ryan Martin, and Carl Schlichting.

\section{BOX 9.2 SUGGESTIONS FOR FUTURE RESEARCH}

- Identify any molecular or otherwise evolutionary signature(s) of plasticity-led evolution that differentiate it from mutation-led evolution. A key impediment to expanding the plasticity-led evolution research program is that the methods traditionally used to study it (see above) may not apply to many taxa or traits. Laboratory experiments and/or additional modeling efforts could be used to identify a signature of plasticity-led evolution that can be applied to diverse taxa, and more importantly, that rules out alternative evolutionary explanations.

- Clarify how different developmental processes, sources of genetic variation, and plasticity mechanisms influence the mode and tempo of plasticity-led evolution. Current efforts have focused primarily on gathering any evidence of plasticity-led evolution. A next step involves comparisons among traits, taxa, and developmental mechanisms to determine if, and how, their propensity to evolve via plasticity-led evolution differs. Comparisons among so-called exploratory processes and other mechanisms of plasticity will likely be fruitful.

- Determine whether particular traits or developmental processes are more or less interchangeable between genetic and environmental control than other such categories. What types of genetic architecture, features of gene regulatory networks, and/or what other aspects of phenotype development make some traits more or less likely to undergo shifts in primary control of development?

- Evaluate under what conditions, if any, polyphenism promotes speciation and identify the mechanisms by which it does so. Polyphenisms have long been theorized to promote speciation and diversification, but there is little direct empirical evidence supporting this idea. Clarifying how speciation via polyphenism differs from other models of speciation, if at all, would be useful.

- Clarify whether and how ancestral plasticity influences the nature of phenotypic diversity during adaptive radiations. Do plastic responses 
tend to bias the phenotypes that arise via radiation? Are plastic traits more likely to drive radiations than non-plastic traits?

- Continue evaluating plasticity-led evolution in diverse traits and taxa.

\section{REFERENCES}

Allf, B. C., P. A. P. Durst, and D. W. Pfennig. 2016. Behavioral plasticity and the origins of novelty: The evolution of the rattlesnake rattle. The American Naturalist 188:475-483.

Aubret, F. and R. Shine. 2009. Genetic assimilation and the postcolonization erosion of phenotypic plasticity in Island tiger snakes. Current Biology 19:1932-1936.

Badyaev, A. V. 2005. Stress-induced variation in evolution: From behavioural plasticity to genetic assimilation. Proceedings of the Royal Society B 272:877-886.

Badyaev, A. V. 2009. Evolutionary significance of phenotypic accommodation in novel environments: An empirical test of the Baldwin effect. Philosophical Transactions of the Royal Society of London, Series B, Biological Sciences 364:1125-1141.

Badyaev, A. V., G. E. Hill, M. L. Beck, A. A. Dervan, R. A. Duckworth, K. J. McGraw, P. M. Nolan, and L. A. Whittingham. 2002. Sex-biased hatching order and adaptive population divergence in a passerine bird. Science 295:316-318.

Badyaev, A. V., A. L. Potticary, and E. S. Morrison. 2017. Most colorful example of genetic assimilation? Exploring the evolutionary destiny of recurrent phenotypic accommodation. The American Naturalist 190:266-280.

Baldwin, J. M. 1896. A new factor in evolution. The American Naturalist 30:441-451.

Bateman, K. 1959. The genetic assimilation of four venation phenocopies. Journal of Genetics 56:443-474.

Bateson, P. 2004. The active role of behaviour in evolution. Biology and Philosophy 19:283-298.

Berrigan, D. and S. M. Scheiner. 2004. Modeling the evolution of phenotypic plasticity, pp. 82-97. In T. J. DeWitt and S. M. Scheiner, eds., Phenotypic plasticity: Functional and Conceptual approaches. Oxford University Press, New York.

Bock, D. G., M. B. Kantar, C. Caseys, R. Matthey-Doret, and L. H. Rieseberg. 2018. Evolution of invasiveness by genetic accommodation. Nature Ecology and Evolution 2:991-999.

Bonduriansky, R. and T. Day. 2018. Extended heredity: A New Understanding of Inheritance and evolution. Princeton University Press, Princeton, NJ.

Carroll, S. B. 2008. Evo-devo and an expanding evolutionary synthesis: A genetic theory of morphological evolution. Cell 134:25-36.

Casasa, S. and A. P. Moczek. 2018. The role of ancestral phenotypic plasticity in evolutionary diversification: Population density effects in horned beetles. Animal Behaviour 137:53-61.

Chan, Y. F., M. E. Marks, F. C. Jones, G. Villareal Jr., M. D. Shapiro, S. D. Brady, A. M. Southwick, D. M. Absher, J. Grimwood, J. Schmultz, R. M. Myers, D. Petrov, B. Jónsson, D. Schluter, M. A. Bell, and D. M. Kingsley. 2010. Adaptive evolution of pelvic reduction in sticklebacks by recurrent deletion of Pitxl enhancer. Science 327:302-305.

Charlesworth, B. 2020. How long does it take to fix a favorable mutation, and why should we care? The American Naturalist 195:753-771.

Chenard, K. and R. A. Duckworth. 2021. The special case of behavioral plasticity? In D. W. Pfennig ed., Phenotypic Plasticity and Evolution: Causes, Consequences, Controversies. CRC Press, Boca Raton, FL.

Conant, G. C. and K. H. Wolfe. 2008. Turning a hobby into a job: How duplicated genes find new functions. Nature Reviews Genetics 9:938-950. 
Corl, A., K. Bi, C. Luke, A. S. Challa, A. J. Stern, B. Sinervo, and R. Nielsen. 2018. The genetic basis of adaptation following plastic changes in coloration in a novel environment. Current Biology 28:2970-2977.

Crispo, E. 2007. The Baldwin effect and genetic assimilation: Revisiting two mechanisms of evolutionary change mediated by phenotypic plasticity. Evolution 61:2469-2479.

Czypionka, T., D. J. Goedbloed, S. Steinfartz, and A. W. Nolte. 2018. Plasticity and evolutionary divergence in gene expression associated with alternative habitat use in larvae of the European Fire Salamander. Molecular Ecology 27:2698-2713.

de Jong, G. 2005. Evolution of phenotypic plasticity: Patterns of plasticity and the emergence of ecotypes. New Phytologist 166:101-117.

Diamond, S. E. and R. A. Martin. 2021. Buying time: Plasticity and population persistence. In D. W. Pfennig, ed., Phenotypic Plasticity and Evolution: Causes, Consequences, Controversies. CRC Press, Boca Raton, FL.

Diggle, P. K. and J. S. Miller. 2013. Developmental plasticity, genetic assimilation, and the evolutionary diversification of sexual expression in Solanum. American Journal of Botany 100:1050-1060.

Ducatez, S., D. Sol, F. Sayol, and L. Lefebvre. 2020. Behavioural plasticity is associated with reduced extinction risk in birds. Nature Ecology \& Evolution 4:1-6.

Duckworth, R. A. 2009. The role of behavior in evolution: A search for mechanism. Evolutionary Ecology 23:513-531.

Ehrenreich, I. M. and D. W. Pfennig. 2016. Genetic assimilation: A review of its potential proximate causes and evolutionary consequences. Annals of Botany 117:769-779.

Fanti, L., L. Piacentini, U. Cappucci, A. M. Casale, and S. Pimpinelli. 2017. Canalization by selection of de novo induced mutations. Genetics 206:1995-2006.

Forsman, A. 2014. Rethinking phenotypic plasticity and its consequences for individuals, populations and species. Heredity 115:276-284.

Foster, S. A. 1994. Inference of evolutionary pattern: Diversionary displays of three-spined sticklebacks. Behavioral Ecology 5:114-121.

Fox, R. J., J. M. Donelson, C. Schunter, T. Ravasi, and J. D. Gaitán-Espitia. 2019. Beyond buying time: The role of plasticity in phenotypic adaptation to rapid environmental change. Philosophical Transactions of the Royal Society B-Biological Sciences 374:20180174.

Futuyma, D. J. 2015. Can modern evolutionary theory explain macroevolution? pp. 29-85. In E. Serrelli, and N. Gontier, eds., Macroevolution: Explanation, Interpretation and Evidence. Springer, New York.

Futuyma, D. J. 2017. Evolutionary biology today and the call for an extended synthesis. Interface Focus 7:20160145.

Futuyma, D. J. 2021. How does phenotypic plasticity fit into evolutionary theory? In D. W. Pfennig, ed., Phenotypic Plasticity and Evolution: Causes, Consequences, Controversies. CRC Press, Boca Raton, FL.

Futuyma, D. J. and M. Kirkpatrick. 2017. Evolution, 4th edition. Sinauer, Sunderland, MA.

Garland Jr., T. and M. R. Rose, eds., 2009. Experimental evolution: Concepts, methods, and applications of selection experiments. University of California Press, Berkeley, CA.

Ghalambor, C. K., K. L. Hoke, E. W. Ruell, E. K. Fischer, D. N. Reznick, and K. A. Hughes. 2015. Non-adaptive plasticity potentiates rapid adaptive evolution of gene expression in nature. Nature 525:372-375.

Gibert, J.-M. 2017. The flexible stem hypothesis: Evidence from genetic data. Development Genes and Evolution 227:297-307.

Gibson, G. and I. Dworkin. 2004. Uncovering cryptic genetic variation. Nature Reviews Genetics 5:681-690.

Glazier, A. M., J. H. Nadeau, and T. J. Aitman. 2002. Finding genes that underlie complex traits. Science 298:2345-2349. 
Goldstein, I. and I. M. Ehrenreich. 2021. Genetic variation in phenotypic plasticity. In D. W. Pfennig, ed., Phenotypic Plasticity and Evolution: Causes, Consequences, Controversies. CRC Press, Boca Raton, FL.

Gomez-Mestre, I. and D. R. Buchholz. 2006. Developmental plasticity mirrors differences among taxa in spadefoot toads linking plasticity and diversity. Proceedings of the National Academy of Sciences of the United States of America 103:19021-19026.

Heil, M., S. Greiner, H. Meimberg, R. Krüger, J.-L. Noyer, G. Heubl, K. E. Linsenmair, and W. Boland. 2004. Evolutionary change from induced to constitutive expression of an indirect plant resistance. Nature 430:205-208.

Hendry, A. P. 2016. Key questions on the role of phenotypic plasticity in eco-evolutionary dynamics. Journal of Heredity 107:25-41.

Hoekstra, H. E., R. J. Hirschmann, R. A. Bundley, P. A. Insel, and J. P. Crossland. 2006. A single amino acid mutation contributes to adaptive beach mouse color pattern. Science 313:101-104.

Hua, J., D. K. Jones, B. M. Mattes, R. D. Cothran, R. A. Relyea, and J. T. Hoverman. 2015. The contribution of phenotypic plasticity to the evolution of insecticide tolerance in amphibian populations. Evolutionary Applications 8:586-596.

Huey, R. B., P. E. Hertz, and B. Sinervo. 2003. Behavioral drive versus behavioral inertia in evolution: A null model approach. The American Naturalist 161:357-366.

Jackson, I. S. and G. E. Budd. 2017. Intraspecific morphological variation of Agnostus pisiformis, a Cambrian Series 3 trilobite-like arthropod. Lethaia 50:467-485.

Jones, B. M. and G. E. Robinson. 2018. Genetic accommodation and the role of ancestral plasticity in the evolution of insect eusociality. The Journal of Experimental Biology 221(23):jeb153163.

Kirschner, M. W. 1992. Evolution of the cell, pp. 99-126. In P. R. Grant and H. S. Horn, eds., Molds, Molecules, and Metazoa: Growing Points in Evolutionary Biology. Princeton University Press, Princeton, NJ.

Kirschner, M. and J. Gerhart. 1998. Evolvability. Proceedings of the National Academy of Sciences USA 95:8420-8427.

Koch, R., A. Kupczok, K. Stucken, J. Ilhan, K. Hammerschmidt, and T. Dagan. 2017. Plasticity first: Molecular signatures of a complex morphological trait in filamentous cyanobacteria. BMC Evolutionary Biology 17:209.

Kovaka, K. 2019. Underdetermination and evidence in the developmental plasticity debate. The British Journal for the Philosophy of Science 70:127-152.

Lamarck, J.-B. 1809. Philosophie zoologique, p. 456.

Lande, R. 2014. Evolution of phenotypic plasticity and environmental tolerance of a labile quantitative character in a fluctuating environment. Journal of Evolutionary Biology 27:866-875.

Ledón-Rettig, C. C. and E. J. Ragsdale. 2021. Physiological mechanisms and the evolution of plasticity. In D. W. Pfennig, ed., Phenotypic Plasticity and Evolution: Causes, Consequences, Controversies. CRC Press, Boca Raton, FL.

Ledón-Rettig, C. C., D. W. Pfennig, and E. J. Crespi. 2010. Diet and hormone manipulations reveal cryptic genetic variation: Implications for the evolution of novel feeding strategies. Proceedings of the Royal Society B: Biological Sciences 277:3569-3578.

Ledón-Rettig, C. C., D. W. Pfennig, A. J. Chunco, and I. Dworkin. 2014. Cryptic genetic variation in natural populations: A predictive framework. Integrative and Comparative Biology 54:1-11.

Lema, S. C. and G. A. Nevitt. 2006. Testing an ecophysiological mechanism of morphological plasticity in pupfish and its relevance to conservation efforts for endangered Devils Hole pupfish. The Journal of Experimental Biology 209:3499-3509.

Levis, N. A. and D. W. Pfennig. 2016. Evaluating 'plasticity-first' evolution in nature: Key criteria and empirical approaches. Trends in Ecology \& Evolution 31:563-574. 
Levis, N. A. and D. W. Pfennig. 2019. Plasticity-led evolution: Evaluating the key prediction of frequency-dependent adaptation. Proceedings of the Royal Society B 286:20182754.

Levis, N. A. and D. W. Pfennig. 2020. Plasticity-led evolution: A survey of developmental mechanisms and empirical tests. Evolution and Development 22:71-87.

Levis, N. A., A. J. Isdaner, and D. W. Pfennig. 2018. Morphological novelty emerges from pre-existing phenotypic plasticity. Nature Ecology and Evolution 2:1289-1297.

Lister, A. M. 2014. Behavioural leads in evolution-evidence from the fossil record. Biological Journal of the Linnean Society 112:315-331.

Losos, J. B. 2014. What is evolution? pp. 3-9. In J. B. Losos, ed., The Princeton Guide to Evolution. Princeton University Press, Princeton, NJ.

Losos, J. B., T. R. Jackman, A. Larson, K. de Queiroz, and L. Rodriguez-Schettino. 1998. Contingency and determinism in replicated adaptive radiations of island lizards. Science 279:2115-2118.

Losos, J. B., D. A. Creer, D. Glossip, R. Goellner, A. Hampton, G. Roberts, N. Haskell, P. Taylor, and J. Ettling. 2000. Evolutionary implications of phenotypic plasticity in the hindlimb of the lizard Anolis sagrei. Evolution 54:301-305.

Martin, C. H., J. E. Crawford, B. J. Turner, L. H. Simons, and C. H. Martin. 2016. Diabolical survival in Death Valley: Recent pupfish colonization, gene flow and genetic assimilation in the smallest species range on earth. Proceedings of the Royal Society B 283:20152334.

Masel, J., O. D. King, and H. Maughan. 2007. The loss of adaptive plasticity during long periods of environmental stasis. The American Naturalist 169:38-46.

Matzkin, L. M. 2012. Population transcriptomics of cactus host shifts in Drosophila mojavensis. Molecular Ecology 21:2428-2439.

Matzkin, L. M., T. D. Watts, B. G. Bitler, C. a. Machado, and T. A. Markow. 2006. Functional genomics of cactus host shifts in Drosophila mojavensis. Molecular Ecology 15:4635-4643.

Mayr, E. 1963. Animal Species and Evolution. Harvard University Press, Cambridge, MA.

McCairns, R. J. S. and L. Bernatchez. 2010. Adaptive divergence between freshwater and marine sticklebacks: Insights into the role of phenotypic plasticity from an integrated analysis of candidate gene expression. Evolution 64:1029-1047.

Mcguigan, K., N. Nishimura, M. Currey, D. Hurwit, and W. A. Cresko. 2011. Cryptic genetic variation and body size evolution in threespine stickleback. Evolution 65:1203-1211.

Messer, P. W. and D. A. Petrov. 2013. Population genomics of rapid adaptation by soft selective sweeps. Trends in Ecology \& Evolution 28:659-669.

Meyer, A. 1993. Trophic polymorphisms in cichlid fish: Do they represent intermediate steps during sympatric speciation and explain their rapid adaptive radiation? pp. 257-266. In J.-H. Schroder, ed., New Trends in Ichthyology. Blackwell, Oxford.

Miller, S., R. Longley, P. Hutchins, and T. Bauersachs. 2020. Cellular innovation of the cyanobacterial heterocyst by the adaptive loss of plasticity. Current Biology 30:344-350.

Moczek, A. P., S. Sultan, S. Foster, C. Ledón-Rettig, I. Dworkin, H. F. Nijhout, E. Abouheif, and D. W. Pfennig. 2011. The role of developmental plasticity in evolutionary innovation. Proceedings of the Royal Society B 278:2705-2713.

Morgan, C. L. 1896. Habit and Instinct. Edward Arnold, London.

Murren, C. J., J. R. Auld, H. Callahan, C. K. Ghalambor, C. A. Handelsman, M. A. Heskel, J. G. Kingsolver, H. J. Maclean, J. Masel, H. Maughan, D. W. Pfennig, R. A. Relyea, S. Seiter, E. Snell-Rood, U. K. Steiner, and C. D. Schlichting. 2015. Constraints on the evolution of phenotypic plasticity: Limits and costs of phenotype and plasticity. Heredity 115:293-301.

Nijhout, H. F. 2003. Development and evolution of adaptive polyphenisms. Evolution and Development 5:9-18. 
Noble, D. W., R. Radersma, and T. Uller. 2019. Plastic responses to novel environments are biased towards phenotype dimensions with high additive genetic variation. Proceedings of the National Academy of Sciences 116:13452-13461.

Orr, H. A. 1999. An evolutionary dead end? Science 285:343-344.

Osborn, H. F. 1896. A mode of evolution requiring neither natural selection nor the inheritance of acquired characters. Transactions of the New York Academy of Sciences 15:141-142.

Paaby, A. B. and M. V. Rockman. 2014. Cryptic genetic variation: Evolution's hidden substrate. Nature reviews Genetics 15:247-258.

Parsons, K. J., K. McWhinnie, N. Pilakouta, and L. Walker. 2020. Does phenotypic plasticity initiate developmental bias? Evolution and Development 22:56-70.

Parsons, K. J., M. Concannon, D. Navon, J. Wang, I. Ea, K. Groveas, C. Campbell, and R. C. Albertson. 2016. Foraging environment determines the genetic architecture and evolutionary potential of trophic morphology in cichlid fishes. Molecular Ecology 25:6012-6023.

Payne, J. L., J. H. Moore, and A. Wagner. 2014. Robustness, evolvability, and the logic of genetic regulation. Artificial Life 20:111-126.

Pennisi, E. 2018. Buying time. Science 362:988-991.

Pfennig, D. W. 2021. Key questions about phenotypic plasticity. In D. W. Pfennig, ed., Phenotypic Plasticity and Evolution: Causes, Consequences, Controversies. CRC Press, Boca Raton, FL.

Pfennig, D. W. and M. McGee. 2010. Resource polyphenism increases species richness: A test of the hypothesis. Philosophical Transactions of the Royal Society B: Biological Sciences 365:577-591.

Pfennig, D. W. and K. S. Pfennig. 2012. Evolution's Wedge: Competition and the Origins of Diversity. University of California Press, Berkeley, CA.

Pfennig, D. W. and A. M. Rice. 2007. An experimental test of character displacement's role in promoting postmating isolation between conspecific populations in contrasting competitive environments. Evolution 61:2433-2443.

Pfennig, D. W., M. A. Wund, E. C. Snell-Rood, T. Cruickshank, C. D. Schlichting, and A. P. Moczek. 2010. Phenotypic plasticity's impacts on diversification and speciation. Trends in Ecology \& Evolution 25:459-467.

Pigliucci, M., K. Cammell, and J. Schmitt. 1999. Evolution of phenotypic plasticity a comparative approach in the phylogenetic neighbourhood of Arabidopsis thaliana. Journal of Evolutionary Biology 12:779-791.

Pigliucci, M., C. J. Murren, and C. D. Schlichting. 2006. Phenotypic plasticity and evolution by genetic assimilation. Journal of Experimental Biology 209:2362-2367.

Price, T. D., A. Qvarnstrom, and D. E. Irwin. 2003. The role of phenotypic plasticity in driving genetic evolution. Proceedings of the Royal Society B: Biological Sciences 270:1433-1440.

Queitsch, C., T. A. Sangster, and S. Lindquist. 2002. Hsp90 as a capacitor of phenotypic variation. Nature 417:618-624.

Robinson, B. W. 2013. Evolution of growth by genetic accommodation in Icelandic freshwater stickleback. Proceedings of the Royal Society B: Biological Sciences 280:20132197.

Rohner, N., D. F. Jarosz, J. E. Kowalko, M. Yoshizawa, W. R. Jeffery, R. L. Borowsky, S. Lindquist, and C. J. Tabin. 2013. Cryptic variation in morphological evolution: HSP90 as a capacitor for loss of eyes in cavefish. Science 342:1372-1375.

Rutherford, S. L. and S. Lindquist. 1998. Hsp90 as a capacitor for morphological evolution. Nature 396:336-342.

Santos, M. E., A. Le Bouquin, A. J. J. Crumière, and A. Khila. 2017. Taxon-restricted genes at the origin of a novel trait allowing access to a new environment. Science 358:386-390.

Scheiner, S. M. 2014. The genetics of phenotypic plasticity. XIII. Interactions with developmental instability. Ecology and Evolution 4:1347-1360. 
Scheiner, S. M. and N. A. Levis. 2021. The loss of phenotypic plasticity via natural selection: Genetic assimilation. In D. W. Pfennig, ed., Phenotypic Plasticity and Evolution: Causes, Consequences, Controversies. CRC Press, Boca Raton, FL.

Scheiner, S. M., M. Barfield, and R. D. Holt. 2017. The genetics of phenotypic plasticity. $\mathrm{XV}$. Genetic assimilation, the Baldwin effect, and evolutionary rescue. Ecology and Evolution 7:8788-8803.

Schlichting, C. D. 2004. The role of phenotypic plasticity in diversification, pp. 191-200. In T. J. DeWitt and S. M. Scheiner, eds., Phenotypic Plasticity: Functional and Conceptual Approaches. Oxford University Press, New York.

Schlichting, C. D. 2021. Plasticity and evolutionary theory: Where we are and where we should be going. In D. W. Pfennig, ed., Phenotypic Plasticity and Evolution: Causes, Consequences, Controversies. CRC Press, Boca Raton, FL.

Schlichting, C. D. and M. Pigliucci. 1998. Phenotypic Evolution: A Reaction Norm Perspective. Sinauer, Sunderland, MA.

Schlichting, C. D. and M. A. Wund. 2014. Phenotypic plasticity and epigenetic marking: An assessment of evidence for genetic accommodation. Evolution 68:656-672.

Schlosser, G. and G. P. Wagner. 2004. Modularity in Development and Evolution. University of Chicago Press, Chicago, IL.

Schluter, D. 2000. The Ecology of Adaptive Radiation. Oxford University Press, Oxford, U. K.

Schluter, D. and L. M. Nagel. 1995. Parallel speciation by natural selection. The American Naturalist 146:292-301.

Schmalhausen, I. I. 1949. Factors of Evolution: The Theory of Stabilizing Selection. University of Chicago Press, Chicago, IL.

Schneider, R. F. and A. Meyer. 2017. How plasticity, genetic assimilation and cryptic genetic variation may contribute to adaptive radiations. Molecular Ecology 26:330-350.

Schwander, T. and O. Leimar. 2011. Genes as leaders and followers in evolution. Trends in Ecology and Evolution 26:143-151.

Scoville, A. G. and M. E. Pfrender. 2010. Phenotypic plasticity facilitates recurrent rapid adaptation to introduced predators. Proceedings of the National Academy of Sciences of the United States of America 107:4260-4263.

Shapiro, A. M. 1975. Genetics, environment, and subspecies differences: The case of Polites sabuleti (Lepidoptera: Hesperiidae). Great Basin Naturalist 35:33-38.

Shapiro, A. M. 1981. Phenotypic plasticity in temperate and subarctic Nymphalis antiopa (Nymphalidae): Evidence for adaptive canalization. Journal of the Lepidopterists' Society 35:124-131.

Shaw, K. A., M. L. Scotti, and S. A. Foster. 2007. Ancestral plasticity and the evolutionary diversification of courtship behaviour in threespine sticklebacks. Animal Behaviour 73:415-422.

Simpson, G. G. 1953a. The Baldwin effect. Evolution 7:110-117.

Simpson, G. G. 1953b. The Major Features of Evolution. Columbia University Press, New York.

Slabbekoorn, H. 2013. Songs of the city: Noise-dependent spectral plasticity in the acoustic phenotype of urban birds. Animal Behaviour 85:1089-1099.

Smith, T. B. and S. Skúlason. 1996. Evolutionary significance of resource polymorphisms in fishes, amphibians, and birds. Annual Review of Ecology, Evolution, and Systematics 27:111-133.

Snell-Rood, E. C. 2012. Selective processes in development: Implications for the costs and benefits of phenotypic plasticity. Integrative and Comparative Biology 52:31-42.

Snell-Rood, E. C., J. D. V. Dyken, T. Cruickshank, M. J. Wade, and A. P. Moczek. 2010. Toward a population genetic framework of developmental evolution: The costs, limits, and consequences of phenotypic plasticity. Bioessays 32:71-81. 
Snell-Rood, E. C., M. E. Kobiela, K. L. Sikkink, and A. M. Shephard. 2018. Mechanisms of plastic rescue in novel environments. Annual Review of Ecology, Evolution, and Systematics 49:331-354.

Sniegowski, P. D. and R. E. Lenski. 1995. Mutation and adaptation: The directed mutation controversy in evolutionary perspective. Annual Review of Ecology and Systematics 26:553-578.

Stein, L. R. and A. M. Bell. 2019. The role of variation and plasticity in parental care during the adaptive radiation of three-spine sticklebacks. Evolution 73:1037-1044.

Susoy, V., E. J. Ragsdale, N. Kanzaki, and R. J. Sommer. 2015. Rapid diversification associated with a macroevolutionary pulse of developmental plasticity. eLife 4:e05463.

Suzuki, Y. and H. F. Nijhout. 2006. Evolution of a polyphenism by genetic accommodation. Science 311:650-652.

Takahashi, M. K. and M. J. Parris. 2008. Life cycle polyphenism as a factor affecting ecological divergence within Notophthalmus viridescens. Oecologia 158:23-34.

Tibbetts, E. A., S. Pandit, and D. Nondorf. 2018. Developmental plasticity and the origin of novel communication systems: Individual recognition in Polistes wasps. Evolution 72:2728-2735.

Torres-Dowdall, J., C. A. Handelsman, D. N. Reznick, and C. K. Ghalambor. 2012. Local adaptation and the evolution of phenotypic plasticity in Trinidadian guppies (Poecilia reticulata). Evolution: International Journal of Organic Evolution 66:3432-3443.

Van Dyken, J. D. and M. J. Wade. 2010. The genetic signature of conditional expression. Genetics 184:557-570.

Waddington, C. H. 1942. Canalization of development and the inheritance of acquired characters. Nature 150:563-565.

Waddington, C. H. 1952. Selection of the genetic basis for an acquired character. Nature 169:278.

Waddington, C. H. 1953. Genetic assimilation of an acquired character. Evolution 7:118-126.

Wcislo, W. T. 1989. Behavioral environments and evolutionary change. Annual Review of Ecology and Systematics 20:137-169.

Weismann, A. 1882. Studies in the Theory of Descent. With notes and additions by the author. In Translated and edited, with notes, by R. Meldola. Sampson, Low, Marston, Searle, \& Rivington, London.

West-Eberhard, M. J. 1989. Phenotypic plasticity and the origins of diversity. Annual Review of Ecology, Evolution, and Systematics 20:249-278.

West-Eberhard, M. J. 2003. Developmental plasticity and evolution. Oxford University Press, New York.

West-Eberhard, M. J. 2021. Foreword: A perspective on 'plasticity'. In D. W. Pfennig, ed., Phenotypic Plasticity and Evolution: Causes, Consequences, Controversies. CRC Press, Boca Raton, FL

Whitehead, A. 2012. Comparative genomics in ecological physiology: Toward a more nuanced understanding of acclimation and adaptation. Journal of Experimental Biology 215:884-891.

Whitehead, A., J. L. Roach, S. Zhang, and F. Galvez. 2011. Genomic mechanisms of evolved physiological plasticity in killifish distributed along an environmental salinity gradient. Proceedings of the National Academy of Sciences of the United States of America 108:6193-6198.

Williams, G. C. 1966. Adaptation and Natural Selection. Princeton University Press, Princeton, NJ.

Wilson, E. O. 1992. The Diversity of Life. Harvard University Press, Cambridge, MA.

Wray, G. A., H. E. Hoekstra, D. J. Futuyma, R. E. Lenski, T. F. C. Mackay, D. Schluter, and J. E. Strassmann. 2014. Does evolutionary theory need a rethink? No, all is well. Nature 514:161-164. 
Wu, R. and M. Lin. 2006. Functional mapping: How to map and study the genetic architecture of dynamic complex traits. Nature Reviews Genetics 7:229-237.

Wund, M. A., J. A. Baker, B. Clancy, J. L. Golub, and S. A. Foster. 2008. A test of the "flexible stem" model of evolution: Ancestral plasticity, genetic accommodation, and morphological divergence in the threespine stickleback radiation. The American Naturalist 172:449-462. 